(C) 2019. This manuscript version is made available under the CC-BY-NC-ND 4.0 license http:// creativecommons.org/licenses/by-nc-nd/4.0/

The definitive publisher version is available online at https://doi.org/10.1016/j.jvcir.2019.102573 


\title{
A New Image Size Reduction Model for an Efficient Visual Sensor Network
}

\author{
Maryam Asadzadeh Kaljahi ${ }^{a}$, Palaiahnakote Shivakumara ${ }^{\mathrm{a}}$, Mohd Yamani Idna Idris ${ }^{\mathrm{a}}$, \\ Mohammad Hossein Anisi ${ }^{\mathrm{b}}$ and Michael Blumenstein ${ }^{\mathrm{c}}$ \\ ${ }^{a}$ Faculty of Computer Science and Information Technology, University of Malaya, Kuala Lumpur, Malaysia \\ ${ }^{b}$ School of Computer Science and Electronic Engineering, University of Essex, Colchester, United Kingdom \\ 'University of Technology Sydney (UTS), Sydney, Australia.
}

asadzadeh@um.edu.my,m.anisi@essex.ac.uk,shiva@um.edu.my, yamani@um.edu.my, michael.blumenstein@uts.edu.au

Corresponding authors: Mohammad Hossein Anisi, Mohd Yamani Idna Idris

\begin{abstract}
Image size reduction for energy-efficient transmission without losing quality is critical in Visual Sensor Networks (VSNs). The proposed method finds overlapping regions using camera locations, which eliminate unfocussed regions from the input images. The sharpness for the overlapped regions is estimated to find the Dominant Overlapping Region (DOR). The proposed model partitions further the DOR into sub-DORs according to capacity of the cameras. To reduce noise effects from the sub-DOR, we propose to perform a Median operation, which results in a Compressed Significant Region (CSR). For non-DOR, we obtain Sobel edges, which reduces the size of the images down to ambinary form. The CSR and Sobel edges of the nonDORs are sent to a VSN. Experimental results and a comparative study with the state-of-the-art methods shows that the proposed model outperforms the existing methods in terms of quality, energy consumption and network life time.
\end{abstract}

Keywords: Visual sensor network; Image size reduction; Inter-redundancy; Intra-redundancy; Energy consumption; Quality of the image.

\section{Introduction}

Wireless sensor networks (WSNs) and visual sensor networks (VSNs) are equipped with low-cost tiny cameras, and play a vital role in real-time applications such as ubiquitous multimedia monitoring applications. However, expanding VSNs to security, wild-life, healthcare or surveillance monitoring is a challenging and interesting task as these applications require a long network life, low energy consumption, cost, etc. Besides, these applications involve several transmissions of images and videos. In addition, as network coverage areas increase, where we can see multiple cameras at multiple locations, the complexity of network resource management increases, significantly. Moreover, the transmission of images and videos requires high energy and network resources (Mammeri et al. 2012; ZainEdin et al. 2015; kaljahi et al. 2018). Therefore, there is immense scope for image size reduction such that a network can use resources efficiently for long life in critical applications. There are numerous methods in the literature to find solutions for network issues, such as energy saving, cost saving and increasing network life (Liu et al. 2016; Navimipour \& Aznoli 2016; Yap \& Yep 2015; Al-Ariki \& Swamy 2016; Shen \& Bai 2016; Bhandary et al. 2016; Lin et al. 2016). Most of the methods focus on network parameters and requirements but not images. In other words, the methods usually use images as a packet for transmission. In addition, VSN consumes more 
energy, time and power for data transmission compared to image acquisition and pre-processing (Lu \& Manduchi 2011; Nirmala et al. 2013; Çağrı Güngör \& Hancke 2013; Ozger et al. 2016). However, the methods do not focus much on removing unwanted information to reduce size for saving energy and power, which lead to decreasing network life-time. This fact motivated us to propose a new image size reduction approach in this work.

There are methods for image size reduction in the literature, which focus on coding, decoding and different compression criteria to reduce the size of the images such that the methods can retrieve the images accurately and efficiently (Peak \& Ko 2015; Rein et al 2016; Sofi \& Naaz 2016; Sastra et al 2015; Yan et al 2018; Yan et al 2014; Yan et al. 2014). However, the methods consider the whole image for processing and hence there is not much reduction in image size. Besides, as size of the image increases, there is potential in losing quality due to the process of compression. Therefore, despite the fact that these methods reduce the size of the image through compression, the information transmitted may not be useful at the base station. Similarly, there are methods, which consider overlapping regions as redundant information to reduce the image size. For example, the methods (Cosar \&Cetin 2015; Imara et al. 2013; O'nils et al. 2012; Imran 2012; Jayashhree et al. 2012) identify the common region with the help of camera fields of view as redundant information. These methods save network energy, time and processing compared to the above methods. However, for complex network systems where many cameras are mounted with different capacities, one can still expect redundant and unwanted information because of the common regions given by multiple cameras. Therefore, there is a need for developing a new method, which can achieve both image size reduction with high quality, but can be independent of the size of the images.

The rest of this paper is organized as follows: Section 2 provides review of the related work. In section 3, we describe the proposed model. In Section 4, we provide experimental results to validate the proposed model. Finally, the conclusion and future work are presented in Section 5.

\section{Related work}

In this section, we review the existing methods on image size reduction for transmission in a VSN environment. The literature on image size reduction can be classified widely as intra-redundancy based methods and inter-redundancy based methods. The intra-redundancy based methods consider the whole image and propose features to identify the region of interest by removing unwanted information in the same image. These methods are effective in simple situations where only one camera is mounted for specific applications. Inter-redundancy based methods consider images captured by multiple cameras and their key common regions as vital information for transmission. These methods are suitable for real time applications where multiple cameras are deployed to monitor the situation in different angles or views. Since the proposed work is aimed at real-time applications such as watching animal behavior in forests, monitoring floods, etc, which requires both intra- and inter-redundancy, the review focuses on both inter- and intraredundancy based methods in this work.

Peak \& Ko (2015) propose k-means clustering-based learning by selecting common colors such that the image size is reduced. However, the method ignores the quality of the images as the size of the image is reduced. Rein et al. (2016) propose a scalable tree-based wavelet image compression algorithm to reduce the size of the image for transmission. Since the method uses a wavelet transform, there are risks of losing significant information due to a blocking effect. In (Sofi \& Naaz 2016) the method proposes several combinations of wavelets for compression to save energy consumption and lifetime of the network. The method does not focus on image quality. Similarly, the work in (Sastra et al. 2015) proposes an improved 
JPEG 2000 compression algorithm that selects optimal parameters such as bit rate of the network and DTW level to reduce energy consumption. However, this method does not change the parameters according to the quality of the image. It is noted from the existing literature that the compression methods developed for the purpose of networking systems, ignore image quality while removing redundant information. In addition, these methods consider the whole image as vital information for transmitting data through the network. Therefore, the methods are expensive and sometimes unfeasible in terms of cost and energy consumption for real-time applications, such as animal surveillance, monitoring floods, garbage, etc.

To overcome the issues of the compression methods, there are other methods which identify the region of interest with the help of feature extraction. The methods transmit only regions of interest rather than transmitting the whole image as in the above methods. For example, in (Redondi et al. 2016) the method uses BRISK features for identifying regions of interest and sends it to a compression algorithm before passing it to the network. These methods work well when we have a priori knowledge of the data. A tracking algorithm is proposed in (Cetin, M., Cosar, S. 2014) and a likelihood function, as significant coefficients are sent to the network, instead of transmission of the whole image, in order to increase the network performance. In (Cosar \& Cetin 2015), the method proposes color and motion features for identifying the regions of interest. These methods are effective when the image contains clear objects. In (Imara et al. 2013; O'nils et al. 2012), the method identifies the region of interest by removing noise and background information. As mentioned earlier, these methods expect clear objects and shapes for achieving accurate results. Similarly, in (Imran 2010), the method considers background subtraction, segmentation and finally Tiff Group4 compression as local processing steps. In (Felemban et al. 2014), the method uses bit planes with most significant bit information for increasing response time. It is observed from the above discussion that these methods may help in reducing the size of the image, while the whole networking system depends on how well the method identifies the region of interest. When using images of floods, and garbage, etc. where there are no specific objects, the methods may not perform well. Further, these methods do not consider the quality of the region of interest.

To alleviate the issues of intra-redundancy based methods for complex situations, there are methods which use inter-redundancy for image size reduction. For example, Jayashhree (2012) proposes a method to detect overlapping regions and non-overlapping regions using spatial information of camera views. Based on the size of the overlapping region, the method chooses an appropriate path to transmit the packet. In other words, the methods gives highest priority to overlapping regions and low priority to non-overlapping regions (Padwalker \& Ambawade 2013). It is noted from experiments that sending the whole overlapping and non-overlapping region on a priority basis is also not effective in terms of networking performance, cost, energy consumption and life-time. These methods focus on the priority to send overlapping and nonoverlapping regions but they do not take full advantage of overlapping and non-overlapping regions. The methods in (Wang et al. 2007 \& 2010) use distortion operations such as the Mean value for the overlapping and non-overlapping regions to reduce the size of the image. The idea is that given the overlapping region is the key region, a distortion operation is applied to reduce the size. At the same time, these methods use different distortion operations for non-overlapping regions because they are not as vital as overlapping regions. Since the method involves a mean operation, which introduces blur during the operation, the images lose quality. In addition, since the window size of the operation is fixed, one cannot expect stable and consistent results. These factors motivated us to propose a new approach for image size reduction to achieve both image size reduction as well as quality. 
The main contributions of this work are three-fold: (i) Finding the dominant overlapping regions that contain useful information by estimating sharpness of the pixels in the images. (ii) Partitioning the dominant overlapping regions further, according to capacity of camera nodes to utilize the resources efficiently. (iii) Proposing a new operation for reducing image size further without losing quality.

\section{Proposed Method}

As noted in the previous sections, we consider multiple cameras with different capacities for data transmission through a Visual Sensor Network (VSN). It is expected that input images can have any size and content. When the system deploys multiple cameras, in general, all cameras focus on particular regions of the images, if the region contains any useful information (Wang et al. 2007 \& 2010). With this notion, the proposed method finds overlapping regions based on camera locations. This helps us to remove unwanted information from the image, which in turn results in image size reduction. To choose the overlapping region that contains useful information, we propose estimated sharpness for the overlapping regions and it finds the region, which gives the highest sharpness compared to other regions in the image as Dominant Overlapping Regions (DORs). If the proposed method does not find overlapping regions, it considers the whole image as input for the image reduction process. Since different cameras have different capacities, to take advantage, the DOR region is further divided into sub-DORs according to the capability and facilities of the cameras, which is called a partition process. To reduce the size of the sub-DOR further, inspired by the method in (Wang et al. $2007 \& 2010$ ) where the Mean operation is used to remove unwanted information from the images, we propose to use a Median operation. This is due to the fact that the Median operation does not introduce blur during the process while the Mean operation introduces blur. This is valid because the Median operation chooses the middle value, which is neither noise (high value) nor nonsignificant pixels (low value) while Mean considers both. The output of the Median operation over subDOR is considered as a Compressed Significant Region (CSR).

The non-DOR is not an important region compared to DOR according to our discussion. However, sometimes, the situation demands other region information along with sub-DORs. Instead of sending the color information, the proposed method sends Sobel edges of non-DORs to the VSN. The main reason to choose the edges is that that the Sobel operator gives fine edges for high contrast information in the image in binary form, which helps in reducing the data. Finally, in the proposed method, CSR with the Sobel of non-DORs are passed to the VSN. In this way, the proposed method achieves image size reduction, along with retaining the quality of the image. The overall flow of the proposed model is illustrated in Fig. 1.

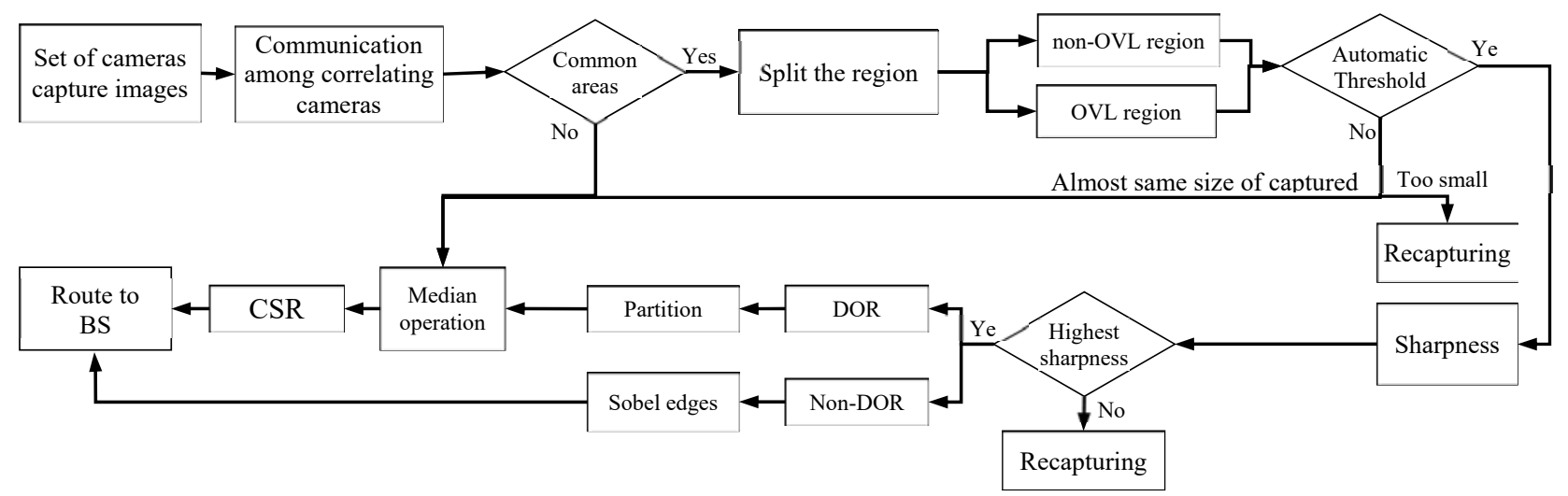

Fig. 1. Block diagram of the proposed method. 


\subsection{OVL and non-OVL Region Detection and Partitioning}

A sample VSN environment can be seen in Fig. 2, where the camera nodes inside the clusters transmit images to a cluster head $(\mathrm{CH})$. The network consists of an $N$ number of camera nodes, say, $C_{i}, i=\{1,2, . ., N\}$ with a predefined initial energy $E_{i} i=\{1,2, . ., N\}$. When camera $C_{i}$ detects an event and captures an image, it broadcasts a query packet to its neighboring camera nodes to find the overlapping region. The responses or communication between cameras indicates that there are overlapping regions. With this networking setup, the proposed model finds overlapping regions by performing an intersection between the areas captured by correlating cameras.

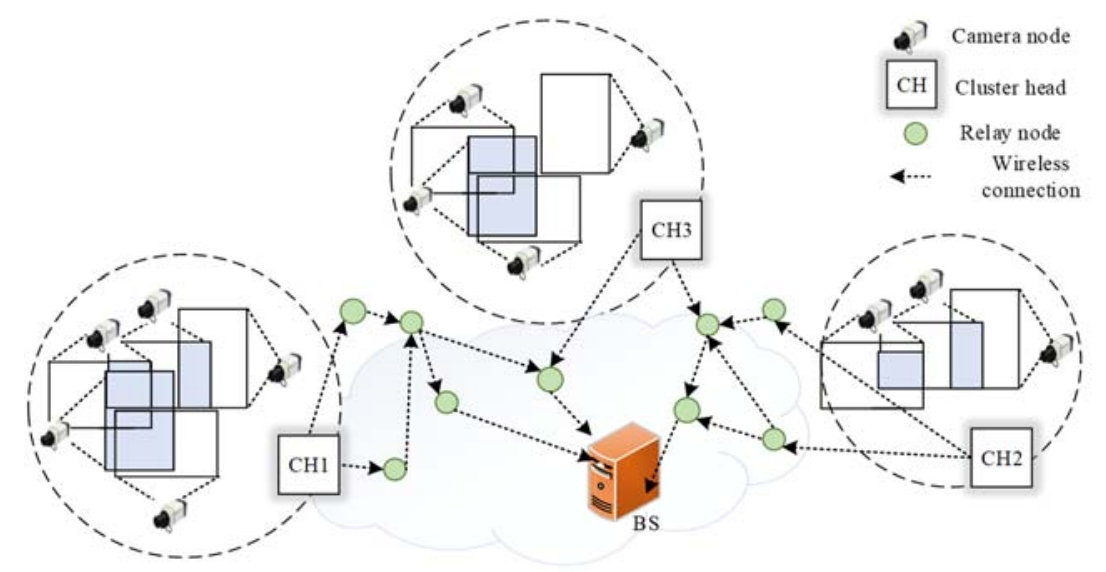

Fig. 2. Sample network system for image transmission.

The process of finding overlapping regions is illustrated in Fig. 3(a) where we can see the nodes are fixed to find overlapping regions from the view of two cameras. Sometimes, the systems give small overlapping regions or big overlapping regions due to different locations and scenarios. If the overlapping region is too small, the proposed method ignores it because it does not contribute. Therefore, we define lower and upper limits for overlapping regions (Costa, et al., 2017). According to the literature and our experiments, if the size of overlapping regions is lower than $10 \%$ of the image captured by a camera, it is considered as being too small and hence it is ignored. Similarly, if the size of the overlapping region is greater than $10 \%$ and less than $50 \%$ of the captured images, it is considered as an actual overlapping region. If the overlapping region is almost the same as the input image size, the proposed method considers the whole image as input for the image reduction process. However, there is no guarantee that the overlapping region provides useful information at all times because the content of overlapping regions vary from one location to another and camera to camera. Therefore, we propose to estimate the sharpness for the overlapping region to highlight the vital information. When two cameras are focusing at the same point (overlapping region), one can expect high sharpness at the overlapping region compared to other regions of the captured images. Based on this observation, we compute the sharpness for the overlapping region as defined in Equation (1). If the sharpness of the overlapping region is higher than other regions of the captured images by the cameras, the proposed method considers it as an actual overlapping region that provides dominant information. If the sharpness of the overlapping region is lower than the other region, the proposed method rejects the overlapping region and images are captured again with the cameras. In this process, the proposed method considers the overlapping region as a window and the same window is moved over captured images as 
shown in the steps in Fig. (3), where (b) and (c) show images captured by camera-1 and camera-2, respectively, and (d) is an overlapping region detected by the camera locations. For visualization, the proposed method performs max-min clustering on the sharpness of the whole image, which classifies high sharpness values into one max cluster and low sharpness values into a min cluster. The output of max clusters of image-1 and image-2 are shown in Fig. 3(e) and Fig. 3 (f), respectively. It is evident from Fig. 3(e) and Fig. 3(f) that the window marked by a green color has more details compared to the windows marked by a red color. This indicates that the overlapping region marked by the green color gives high sharpness compared to the other window marked by a red color as shown in Fig. 4(a). Therefore, we can assert that the overlapping region contains useful or focused information, which is called a Dominant Overlapping Region (DOR).

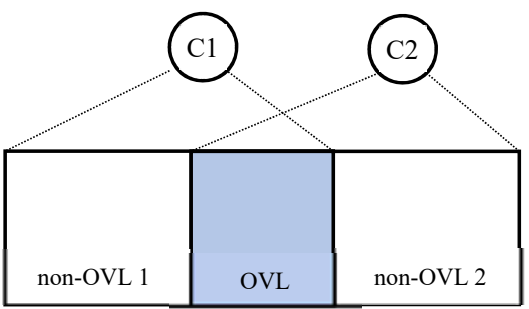

(a) Example of finding an overlapping region using a two-camera set-up

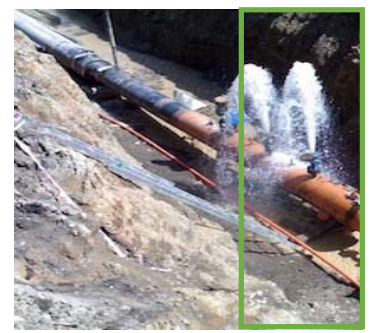

(b) Image captured by $\mathrm{C}_{1}$

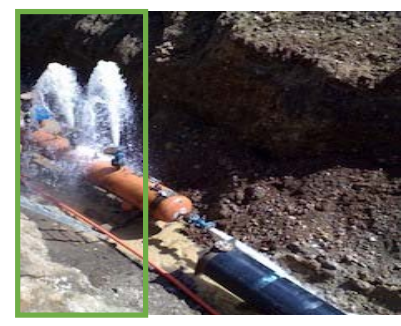

(c) Image captured by $\mathrm{C}_{2}$

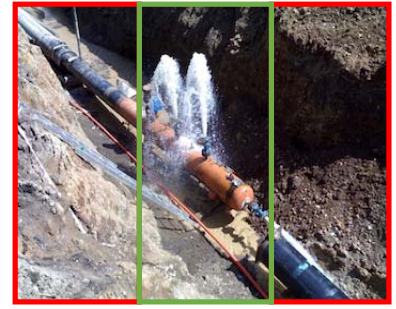

(d) Dominant overlapping region

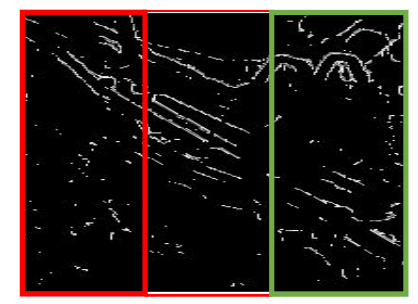

(e) Max cluster sharpness of $\mathrm{C}_{1}(\mathrm{~N} 11, \mathrm{~N} 12, \mathrm{DOR})$

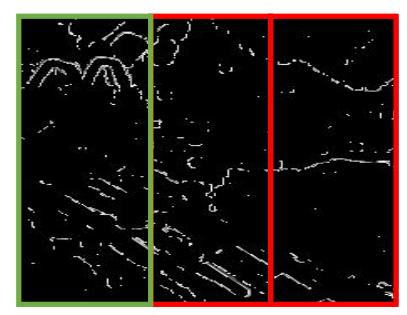

(f) Max cluster sharpness of $\mathrm{C}_{2}(\mathrm{DOR}, \mathrm{N} 21, \mathrm{~N} 22)$

Fig.3. Finding an overlapping region that provides dominant information

N11, N12 denote non-overlapping regions of the $\mathrm{C}_{1}$ image; N21, N22 denote non-overlapping regions of $\mathrm{C}_{2}$.

In Equation (1), $\theta$ denotes the average of the gradient values in the window of size $5 \times 5$, the numerator denotes fourth moments around the mean divided by the square of the second moment around the mean. The offset of 3 is to measure the normal distribution to be zero. The sharpness is estimated as defined in Equation (2) where $\mathrm{G}_{\mathrm{x}}$ and $\mathrm{G}_{\mathrm{y}}$ are gradient magnitudes along the $\mathrm{x}$ and $\mathrm{y}$ directions, respectively. The same offset of 3 is used for defining Equation (1) to avoid a zero effect. Sample overlapping regions that give dominant information for the different images are shown in Fig. 4(b). 


$$
\begin{gathered}
W=\frac{E\left[(G-\theta)^{4}\right]}{E\left[(G-\theta)^{2}\right]}-3 \\
S=\min \left(\operatorname{Ln}\left(W\left(G_{x}\right)+3\right), \operatorname{Ln}\left(W\left(G_{y}\right)+3\right)\right)
\end{gathered}
$$

In order to utilize the resources of each camera node, the proposed model partitions the DOR region further into sub-DORs according to energy levels at camera nodes, as shown in Fig. 5(a), where sub-DOR-2 is a small region requested by camera node $\mathrm{C}_{2}$ and sub-DOR-1 being larger than sub-DOR-2 is requested by another camera node $\mathrm{C}_{1}$. The energy of each camera node is defined in Equation (3). For example, a sample blue colored DOR region is shown in Fig. 5(a) where $C_{1}$ with $E_{1}=45 \mathrm{~J}$ and $C_{2}$ with $E_{2}=15 \mathrm{~J}$ divide the share of the DOR based on the energy proportion defined in Equation (3) where $M$ is the number of correlating cameras. This shows that $75 \%$ of the DOR (sub-DOR-1) are transmitted through $C_{1}$ and $25 \%$ of DOR (sub-DOR-2) are transmitted through $C_{2}$ as shown in Fig. 5(a). The process of partitioning is illustrated in Fig. 5(b) where we can see sub-DOR-1 and sub-DOR-2 are the sub-DORs, which result in a partition, whereas the non-DOR-1 and non-DOR-2 are the non-DORs of the images.

$$
f_{i}=\frac{E_{i}}{\sum_{j=1}^{M} E_{j}}
$$

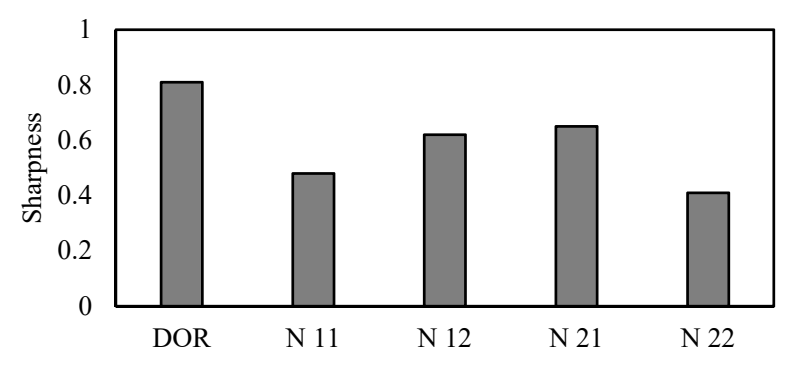

(a) Sharpness of overlapping and non-overlapping windows of the captured images.
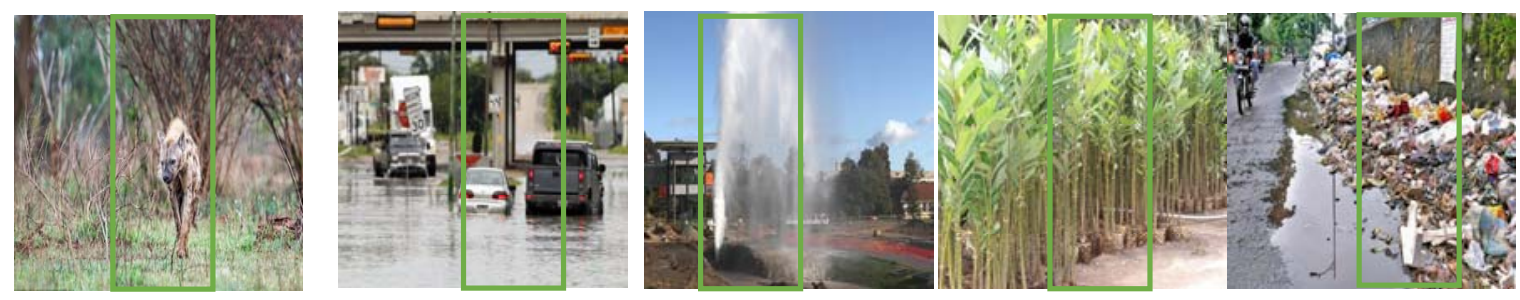

(b) Example of overlapping regions that provide dominant information

Fig. 4. Dominant Overlapping Regions (DORs) based on sharpness. 


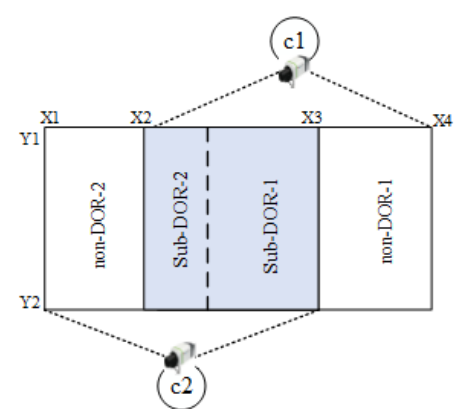

(a)

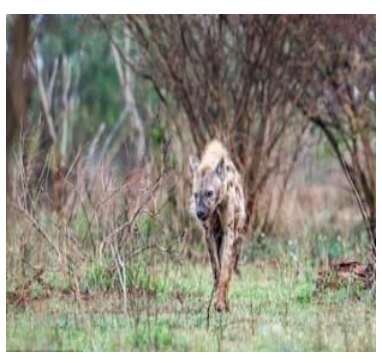

Input image

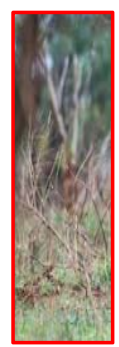

non-OVL-2

(b)

Fig. 5. Sub-DOR and non-DOR after partition.

\subsection{Size Reduction Model for Optimal Transmission}

Sometimes, DORs may still contain unwanted information due to some operations. The proposed method performs a Median operation, which eliminates unwanted information from the images without affecting vital information. In contrast to the Mean operation used in (Wang et al. 2007 \& 2010), the Median operation is better in terms of quality. Therefore, one can say that the Median operation can also reduce the size of the images, which enhances VSN performance further. It is evident from Fig. 6 (a), where it can be observed that as the window size $(T)$ increases, the blurriness increases, at the same time, the number of bytes $(K)$ also decreases. On the other hand, in the case of the Median operation, though the number of bytes decreases, the quality does not decrease as for the Mean operation, as shown in Fig. 6 (b). This shows that the Mean operation is too sensitive to content, but the Median is not. However, it is confirmed that the quality of the image and size of the image depend on the window size.

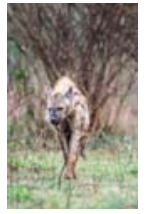

$\mathrm{T}=2$ $\mathrm{k}=0.22$

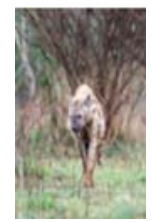

$\mathrm{T}=2$

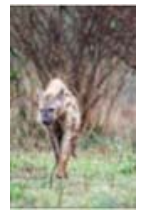

$$
\mathrm{T}=3
$$$$
\mathrm{k}=0.19
$$

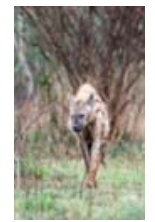

$\mathrm{T}=3$

$\mathrm{k}=0.20$

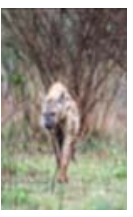

$$
\mathrm{T}=4
$$

$\mathrm{k}=0.16$

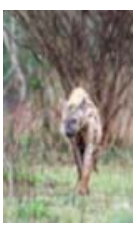

$\mathrm{T}=4$

$\mathrm{k}=0.18$

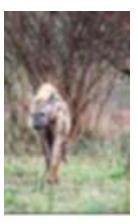

$\mathrm{T}=5$ $\mathrm{k}=0.15$
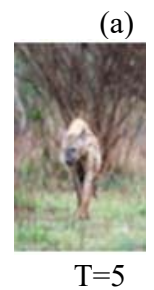

$\mathrm{k}=0.16$

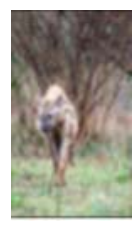

$\mathrm{T}=6$ $\mathrm{k}=0.13$

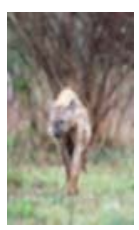

$\mathrm{T}=6$ $\mathrm{k}=0.15$

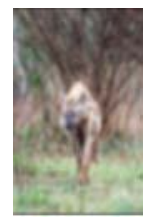

$$
\mathrm{T}=7
$$$$
\mathrm{k}=0.12
$$

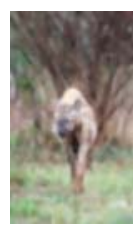

$\mathrm{T}=7$

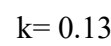

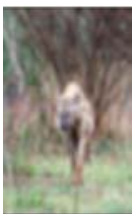

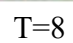

$\mathrm{k}=0.11$

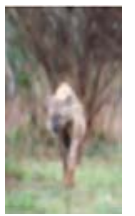

$\mathrm{T}=8$

$\mathrm{k}=0.13$

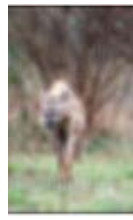

$\mathrm{T}=9$ $\mathrm{k}=0.10$

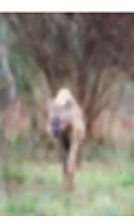

$\mathrm{T}=9$

$\mathrm{k}=0.11$

Fig. 6. The results of Mean (a) and Median (b) operations on a sample DOR image for different windows (T). $\mathrm{K}$ denotes the number of bytes.

To determine window size automatically, we propose a new idea which estimates quality of the DOR by varying the window size. For this, we use standard quality measures, namely PSNR and SSIM as defined in Equation (4) and (5), respectively. Note that to calculate these two measures, we use the original DOR as ground truth and the result of the window operation as processed images. Higher PSNR and SSIM values indicate high quality of the image. This is illustrated in Fig. 7(a) and Fig. 7(b) where for window size T=3, 
both PSNR and SSIM score high values. This shows that window size T=3 is appropriate for the DOR. In this way, the proposed model determines window size automatically.

$$
\begin{aligned}
& \operatorname{PSNR}=10 \times \log _{10}\left(\mathrm{MAX}_{\mathrm{I}}^{2} / \mathrm{MSE}\right) \\
& \operatorname{MSE}=1 / \mathrm{m} \times \mathrm{n} \sum_{\mathrm{i}=0}^{\mathrm{m}-1} \sum_{\mathrm{j}=0}^{\mathrm{n}-1}[\mathrm{P}(\mathrm{i}, \mathrm{j})-\mathrm{K}(\mathrm{i}, \mathrm{j})]^{2} \\
& \operatorname{SSIM}(\mathrm{x}, \mathrm{y})=\frac{\left(2 \mu_{\mathrm{x}} \mu_{\mathrm{y}}+\mathrm{c}_{1}\right) \times\left(2 \delta_{\mathrm{xy}}+\mathrm{c}_{2}\right)}{\left(\mu_{\mathrm{x}}^{2}+\mu_{\mathrm{y}}^{2}+\mathrm{c}_{1}\right) \times\left(\delta_{\mathrm{x}}^{2}+\delta_{\mathrm{y}}^{2}+\mathrm{c}_{2}\right)}
\end{aligned}
$$

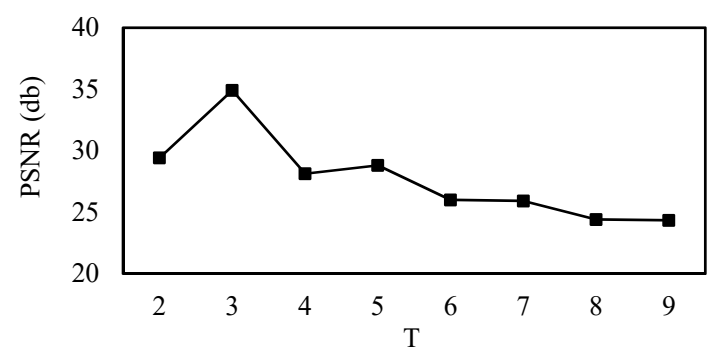

(a) PSNR according to different window size (T)

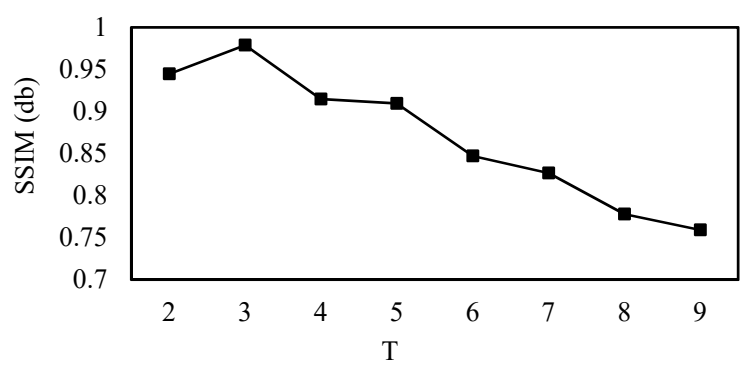

(b) SSIM for different window size (T)

Fig. 7. Illustration for choosing $T$ for image size reduction.

In Equation (4), $M A X_{I}^{2}$ is the maximum possible value of the image's pixels and $M S E$ is the mean square error. $M S E$ is the sum of all squared differences of DOR image $P$ and $K$, as the result of window operations divided by image size. In Equation (5), $\operatorname{SSIM}(x, y)$ is a similarity metric in which $\mu_{x}, \mu_{y}$ and $\delta_{x}^{2}, \delta_{y}^{2}$ are the average and variance of $x$ and $y$ in a window size, while $\delta_{x y}$ denotes their covariance. The proposed method sends the result of the Median operation of DOR to the VSN, which is called the CompressedSignificant Region (CSR). As mentioned in the proposed method section, the non-DOR is not important for the VSN, and thus we send Sobel edges of the non-DOR along with the CSR, as shown in Fig. 8. Where we can see for the sample input image in (a), the CSR given by Median operation with $T=3$ and Sobel edges for the non-DOR are the final data to be sent to the VSN, as shown in Fig. 8(b). When we calculate the total size of the final image according to Equation (6), it gives $8.7 \mathrm{~kb}$, which includes $5.4 \mathrm{~kb}$ of CSR and $3.3 \mathrm{~kb}$ for the Sobel of the non-DOR in contrast to the total size of the original image, which is $37.9 \mathrm{~kb}$. This shows that the proposed model uses the resources of the camera nodes efficiently in terms of image size reduction and quality of the image. In equation (6), $S Z_{\text {total }}$ denotes the total size of the image that will be sent, $S Z_{C S R}$ denotes the size of the processed overlapping region and $S Z_{n o n-D O R}$ denotes the size of the nonoverlapping region.

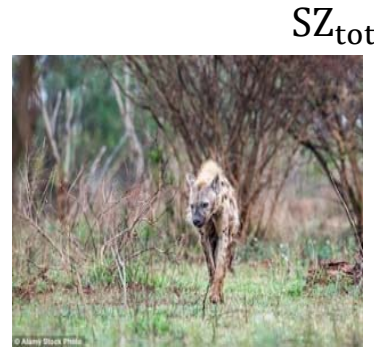

(a) Sample input image (b) Final image sent to VSN (CSR+Sobel of non-DOR) Fig. 8. Compressed significant region with edge information of the non-DOR. 


\section{Experimental results}

Since there is no standard or benchmark dataset for evaluating the performance of the proposed method, we create our own dataset for experimentation in this work. We consider situations, where networking is essential, namely, watching animal behavior in the forest, monitoring flood levels, plant growth in the fields, cracks in the soil or water pipes and cleaning garbage. In total, we consider five classes, whereby each class contains 20 different scene images, which gives 100 images for experimentation. To capture the images, we use a low cost camera with a resolution of $600 \times 450$ pixels. Sample images of the above five classes are shown in Fig.9, where one can guess that the scene images are complex in nature due to variations in background and foreground. The initial energy for each node is set to $E_{i}=50 \mathrm{j}$. Each camera node has an ability to compress the images using a compression algorithm until the energy reaches a minimum threshold $E_{i}=D T$. The distance between the node and the $C H$ is 10 meters.

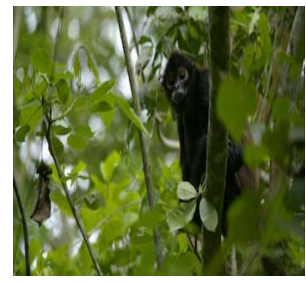

Animal

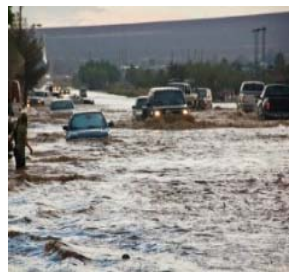

Flood

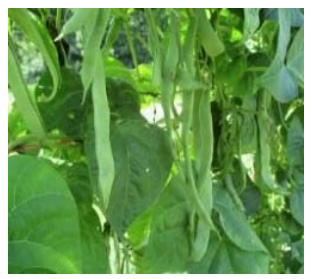

Plant

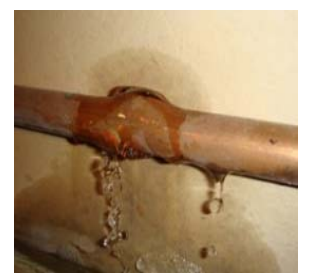

Pipeline

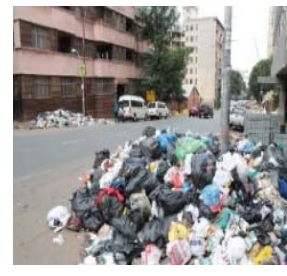

Garbage

Fig. 9. Sample images of five classes for experimentation.

The proposed method comprises two key steps, namely, finding the compressed significant region (CSR) and the use of resources of the camera nodes in the VSN. Therefore, we use standard measures, namely, PSNR and SSIM as mentioned in the previous section for evaluating the quality of the CSR. In the same way, to assess the performance of the networking system, we define the energy model used for communication in an existing method (Sukumaran 2015; Nandgini \& Radha 2016), as defined in Equation (7), where $E_{b i t}$ is the transmission energy per bit, $t$ denotes the transmission time on a $250 \mathrm{kbits} / \mathrm{s}$ IEEE 802.15.4 link, $I$ and $V$ denote the current and voltage of the TelosB node. Accordingly, the computed transmission energy per bit is $0.23 \mu \mathrm{J}$.

$$
\mathrm{E}_{\text {bit }}=\frac{(\mathrm{t} \times \mathrm{I} \times \mathrm{V})}{1024}
$$

We calculate Average Residual Energy (ARE) as defined in Equation (8) and Life Time (LT) as defined in equation (9). $A R E$ is defined as the average residual energy of $N$ nodes in the $n^{\text {th }}$ iteration. This measure helps us to evaluate the camera node efficiency in terms of energy during image transmission. Similarly, $L T$ is defined based on the number of iterations that the whole cluster can capture, the process and the transmission of images before reaching DT. In equation (8) and (9), $r$ denotes the number of scenarios, $E_{i}$ denotes energy at the $i^{\text {th }}$ node and the $n^{\text {th }}$ iteration, Itr is the number of iterations, $t$ is the estimated time for each iteration and $m$ denotes minutes and $h$ denotes hours.

$$
\begin{aligned}
\mathrm{ARE} & =\frac{\sum_{\mathrm{i}=1}^{\mathrm{N}} \mathrm{E}_{\mathrm{i}}}{\mathrm{r}} \\
\mathrm{LT} & =\frac{\mathrm{Itr} \times \mathrm{t}}{\mathrm{m} \times \mathrm{h}}
\end{aligned}
$$

To show the effectiveness of the proposed method, we implement a state-of-the-art method (Wang et al. 2010) to compare with the proposed method in terms of image quality and other networking parameters. 
The reason to choose this method is that the objective of this method is the same as the proposed method. It uses a Mean operation for image size reduction for the overlapping region as in the proposed method. For image size reduction, there are Distributed Source Coding (DSC) systems, which aim at improving the performance of the visual sensor network without losing quality of the images when the image data is huge. In order to show that the proposed method is superior to DSC, we implement DSC as proposed in (Wang et al. 2007). The DSC usually captures images by different sensors and cameras and then use different level of compression according to redundant information in the captured images. As redundancy increases, the performance of the DSC increases, else the performance decreases. However, in this work, we pass 100 images captured by two cameras to DSC for experimentation. The level of compression is determined empirically by experimenting on pre-defined cases (scenarios) chosen randomly for the images in the database. It is expected that as the size of the input images increases, the performance of the DSC decreases in terms of quality, efficiency, energy etc. This is due to more operations involved in compression while distributing image data of a huge size. On the other hand, in our case, the proposed method finds CSR, which passes through the visual sensor networks, and is independent of input size. Therefore, the proposed method works well irrespective of the size of the images.

\subsection{Evaluating the Image Quality with the Median Operation}

To validate the image quality of CSR given by the proposed method and existing methods, we calculate PSNR and SSIM for the output of the methods as shown in Fig. 10(a) and Fig. 10(b), respectively. It is observed from Fig. 10(a) and Fig. 10(b) that the proposed method reports high PSNR and SSIM for all the images compared to the existing methods. This is valid because the method (Wang et al. 2010) use Mean operations with a fixed sized window for removing unwanted information, which introduces blur during the operation. As a result, the existing method loses quality of the image and hence leads to poor PSNR and SSIM. Since the performance of DSC depends on size of the image, as size varies the method loses the quality of the image. Therefore, the method reports low PSNR and SSIM compared to the proposed method. However, when we compare the results of the two existing methods, (Want et al. 2007) is better than the method in (Want et al. 2010). On the other hand, the proposed method performs a Median operation for the sub-DORs given by a common region, sharpness and partition with a dynamic window size, that is why the proposed method reports the best score compared to the existing methods.

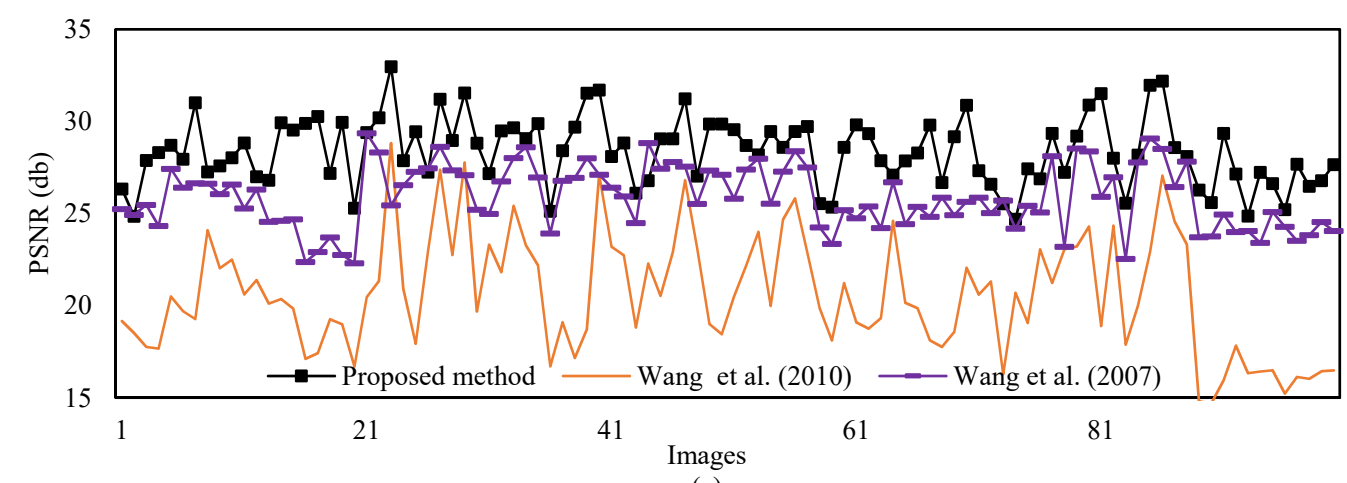

(a) 


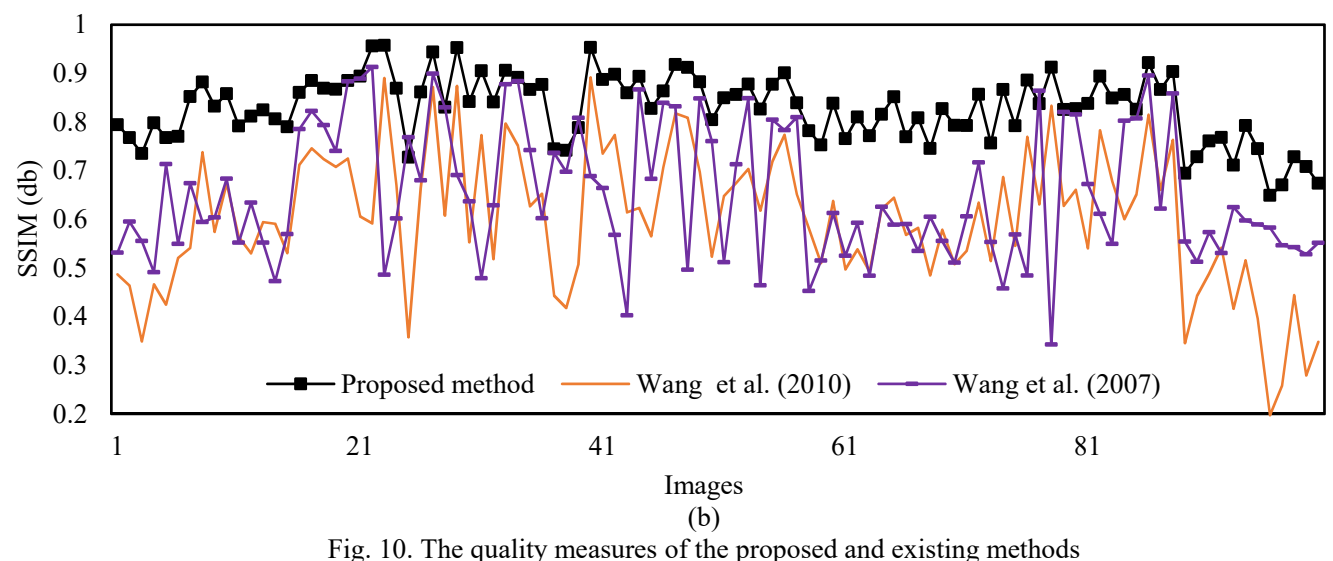

\subsection{Evaluating the Networking System}

For this experiment, we set 100 different scenarios for evaluating the proposed and existing methods in terms of $A R E$ and $L T$. To calculate $A R E$, we consider 100 random scenarios. ARE is then estimated for the $200^{\text {th }}$ iteration of 100 scenarios to evaluate the proposed and existing methods. We use (Wang et al. 2010) for this experiment because this method involves the same steps as the proposed method. The proposed method involves three key steps, namely, finding overlapping regions (OVL), finding sub-Dominant Overlapping region (Sub-DOR) with the partition step, and the last Compressed Significant Region (CSR). To show these steps consume less energy and give a higher life time for the network compared to the whole image, we calculate ARE and LT by passing the whole image, OVL, Sub-DOR and CSR to the VSN as reported in Fig. 11(a) and Fig. 11(b), respectively. It is observed from Fig. 11(a) and Fig. 11(b) that the proposed and existing method report high $A R E$ and $L T$ for the steps compared to the whole image. This

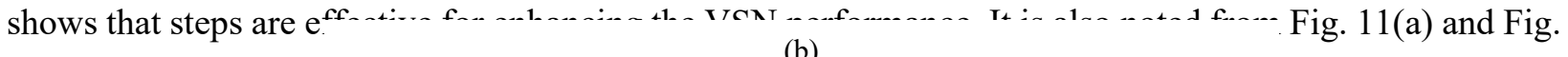
11(b) that the proposi Fig. 12. The quality measures of the proposed and existing methods $\quad: R$ compared to the existing method. Hov. ....., .... r. OVL. This is valid because the methods use the same criteria for finding an overlapping region.

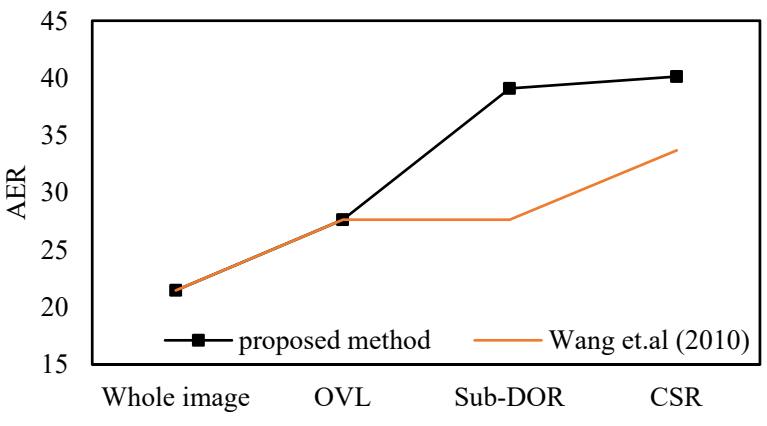

(a)

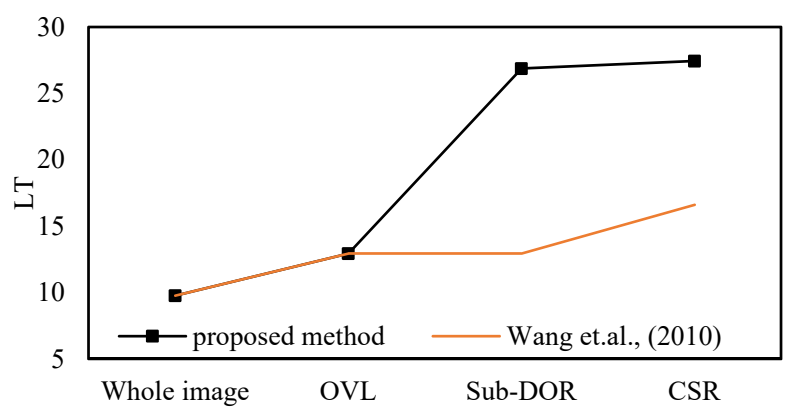

(b)

Fig. 11. Comparing $A E R$ and $L T$ of the proposed system with the existing system in terms of ARE and LT with $N=5$

In addition, we calculate $L T$ for the proposed method and existing methods including DSC by varying the number of cameras $(N=2,3,4,5)$, as shown in Fig. 12(a) where it can be noted that $L T$ is higher for the proposed method compared to both the existing methods. The $L T$ of (Wang et al. 2010) is higher than the DSC (Wang et al. 2007), which is poorer than the proposed method. This is due to complex operations for 
finding compression in the case of DSC compared to a simple Mean operation. It was also found from Fig. 12(a) that the results of the proposed and existing methods increase as the number of cameras increase. This is because in the case of networking, as the number of choices increase, the network systems choose the best one for transmission. The main reasons that the existing method demonstrates poorer performance compared to the proposed method are the same as discussed above.

In this work, we use 5 low cost cameras with a 10 metre distance for simulation. In realtiy, one can expect different set ups for the different situations. It is true that determination of the exact number of cameras for every situation is hard. In order to decide the number of cameras, we should have some knowledge of the location, situation, and condition, etc. In the same way, the distance between the camera and the ground is also an improtant parameter that affects the performance of the VSN as it depends on the area, capacity of the camera nodes and cost. One such illustration is shown in Fig. 12(b), where we can see that as the distance (d) increases, the nubmer of iterations decreases. The proposed and existing methods show a sudden drop at a 70 metre distance. This shows that the resources used in the proposed and existing methods are not enough to handle the situations beyond a 70 metre distance. Therefore, developing a model which is independent of these parameters is interesting and challenging for future research.

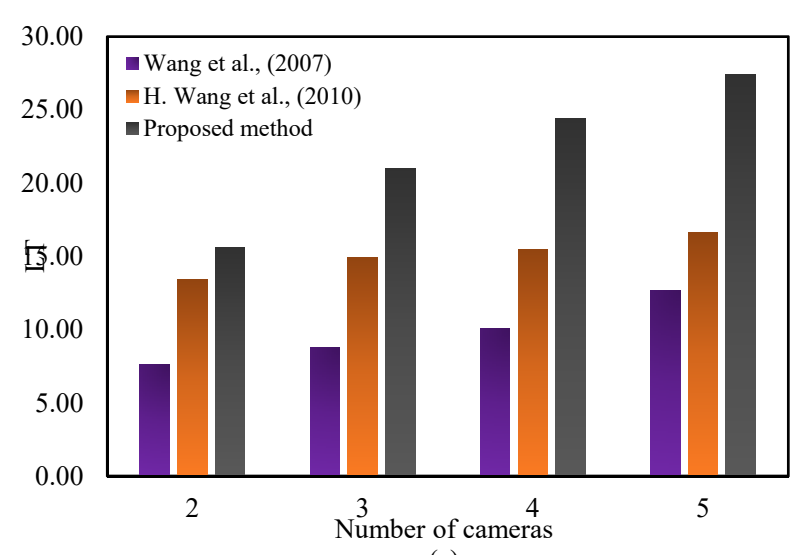

(a)

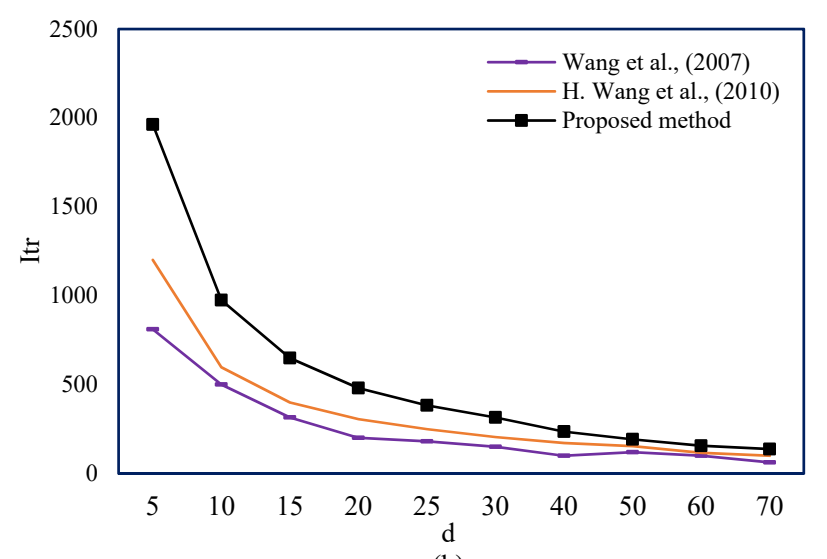

(b)

Fig. 12. Comparing performance of the proposed and existing methods in terms of $L T$ with $N=2,3,4$ and 5 (a) and Itr with different $d$ (b).

\section{Conclusions}

In this paper, we have proposed a new image size reduction model for enhancing the performance of a VSN networking system. The proposed model detects overlapping and non-overlapping regions based on responses and locations. The proposed model introduces a Dominant Overlapping Region (DOR) by analyzing the sharpness of the overlapping region, which confirms the useful information in the overlapping region. The proposed method uses the camera capacities to partition the DOR into sub-DORs to enhance the VSN performance further. Further, the proposed method performs a Median operation over the subDOR to remove any unwanted information, which results in a Compressed Significant Region (CSR). Experimental results on image quality given by the proposed and exiting methods show that the proposed model is better than the existing methods in terms of PSNR and SSIM. Experiments on a networking system show that the proposed model is also better than the existing methods in terms of iterations, energy consumption and network lifetime. As discussed in the experimental section, there is scope for improving the performance of the proposed method, especially improving performance of the whole system when the number of cameras increases and distance between the camera and ground increases. 


\section{Acknowledgments}

This research work was supported by the Faculty of Computer System and Information Technology at the University of Malaya under grant numbers: RP036B-15AET and PG063-2016 A.

\section{References}

Al-Ariki, H. D. E., Swamy, M.N. S. (2016) A survey and analysis of multipath routing protocols in wireless multimedia sensor networks", Wireless Network, 1-13.

Bhandary, V., Malik, A. \& Kumar, S. (2016) Routing in wireless multimedia sensor networks: a survey of existing protocols and open research issues", Journal of Engineering.

Çağrı Güngör, V., Hancke, G. P. (2013) Industrial wireless sensor networks: Applications, protocols, and standards”. Crc Press.

Cetin, M., Cosar, S. (2014) Feature compression: A Framework for multi-view multi-person tracking in visual sensor networks", Journal of visual communication and image representation, 25, 5, 864-873.

Cosar, S., Cetin, M. (2015) Sparsity-driven bandwidth-efficient decentralized tracking in visual sensor networks", Computer Vision and Image Understanding, 139, 40-58.

Costa, D. G., Duran-Faundez, C., and Bittencourt, J. C. N. (2017). Availability issues for relevant area coverage in wireless visual sensor networks. The Electrical, Electronics Engineering, Information and Communication Technologies (CHILECON).

Felemban, E., Sheikh, A. A. \& Manzoor, M. A. (2014) "Improving response time in time critical Visual Sensor Network applications", Ad hoc Networks, 23, 65-79.

Imara, M., Khursheed, Kh., Ahmad, N., O’Nils, M., Lawal, N. \& Waheed, M. A. (2013) Architecture of wireless visual sensor node with region of interest coding", In Proc. NESEA.

Imran, M. (2010) Exploration of target architecture for a wireless camera based sensor node", In Proc. IEEE NORCHIP, 1-4.

Jayashhree, A., Biradar, G. S. \& Mytri, V.D. (2012) Energy efficient prioritized multipath QoS routing over WMSN", Computer Applications, 46.

Lin, K., Rodrigues, J., Ge, H., Xiong, N., Liang, X. (2011) Energy efficiency QoS assurance routing in wireless multimedia sensor networks", IEEE Systems Journal, 5, 4.

Liu, J., Sridharan S., \& Fookes, C. (2016) Recent advances in camera planning for large area surveillance: a comprehensive review", ACM Computing Surveys, 49, 1-37.

Lu, X., Manduchi, R. (2011) Fast image motion segmentation for surveillance applications", Image and Vision Computing, 104116.

M. A. Kaljahi, M. A. P. Shivakumara, M. H. Anisi, M. Y. L. Idris, M. Blumenstein, and M. K. Khan. (2018), A scene image classification technique for a ubiquitous visual surveillance system, Multimedia Tools and Applications, 1-28.

Mammeri, A., Hadjou, B. \& Khoumsi, A. (2012) A Survey of image compression algorithms for visual sensor networks", Sensor Networks.

Nandgini S. A., Radha, S. (2016) "Compressed Sensing based object detection and tracking system using Measurement Selection Process for Wireless Visual Sensor Networks", In Proc. Signal Processing and Networking (WiSPNET).

Navimipour N. J., Aznoli, F. (2016) "Deployment strategies in the wireless sensor networks: systematic literature review, classification, and current trends", Wireless Personal Communications, 1-28, 2016.

Nirmala, D. E., Vignesh, R. K. \& Vaidehi, V. (2013) Multimodal image fusion in visual sensor networks", In Proc. International Conference on Electronics, Computing and Communication, 1-6.

O'nils, M., Khursheed, Kh., Ahmad, N. \& Imran, M. (2012) Detecting and coding region of interests in bi-level images for data reduction in wireless visual sensor network", In Proc. International Conference on Wireless and Mobile Computing, 705712.

Ozger, M., Fadel, E. \& Akan, O. (2016) Event-to-sink spectrum-aware clustering in mobile cognitive radio sensor networks", IEEE Transactions on Mobile Computing, 15, 9, 2221-2233.

Padwalker, U., Ambawade, D.D. (2013) Multipath based energy efficient (MEE) routing protocol for WMSNs", Computer Applications.

Peak J., Ko, J. (2015) K-Means clustering-based data compression scheme for wireless imaging sensor networks", IEEE System Journal, 99, 1-11.

Redondi, A., Baroffio, L., Cesana M. \& Tagliasacchi, M (2016) Compress-then-analyze versus analyze-then-compress :what is best in visual sensor networks", IEEE Transaction on Moble Computing, 15.

Rein, S., Reisslein, M. \& Chen, H. (2016) "Scalable line-based wavelet image coding in wireless sensor networks", Journal of Visual Communication and Image Representation, 40,418-431.

Sastra, N. P., Wirawan, I. \& Hendratoro, G. (2015) Energy efficiency of image compression for virtual view image over wireless visual sensor network", Journal of Networks.

Shen, H., Bai, G., (2016) Routing in wireless multimedia sensor networks: A survey and challenges ahead", Journal of Networkand Computer Applications, 71, 1-49. 
Sofi, S. A., Naaz, R. (2016) “Data compression in wireless visual sensor networks using wavelets”, In Proc. International Conference on Wireless Communications, Signal Processing and Networking (WiSPNET).

Sukumaran, A. N., Sankarajan, R. \& Rajendiran, K. (2015) Video Compressed Sensing framework for Wireless Multimedia Sensor Networks using a combination of multiple matrices", Computers and Electrical Engineering, 44, 51-66.

Wang, D., Peng, H., Wang, W., Sharif H., \& Chen, H., (2010) Image transmissions with security enhancement based on region and path diversity in wireless sensor networks", IEEE Transactions on Wireless Communications, 8, 757-765.

Wang, H., Peng, D., Wang, W., \& Sharif, H. (2007) Collaborative image transmissions based on region and path diversity in wireless sensor network", In Proc. Telecommunication Conference (GLOBCOM), 2007.

Wang, P., Li, C. and Zhen, J. (2007), Distributed data aggregation using clustered slepian-wolf coding in wirelss sensor networks, In Proc. ICC 2007, 3616-3622.

Yan, C., Xie, H., Yang, D., Yin, J., Zhang, Y., \& Dai, Q. (2018). Supervised hash coding with deep neural network for environment perception of intelligent vehicles. IEEE transactions on intelligent transportation systems, 19(1), $284-295$.

Yan, C., Zhang, Y., Xu, J., Dai, F., Li, L., Dai, Q., \& Wu, F. (2014). A highly parallel framework for HEVC coding unit partitioning tree decision on many-core processors. IEEE Signal Processing Letters, 21(5), 573-576.

Yan, C., Zhang, Y., Xu, J., Dai, F., Zhang, J., Dai, Q., \& Wu, F. (2014). Efficient parallel framework for HEVC motion estimation on many-core processors. IEEE Transactions on Circuits and Systems for Video Technology, 24(12), 2077 2089.

Yap G. H., Yen, H. (2015) Novel Visual Sensor Deployment Algorithm in Occluded Wireless Visual Sensor Networks", IEEE Systems Journal, pp. 1-12.

ZainEdin, H., Elhosseini M. A., \& Ali, H. A. (2015) Image compression algorithms in wireless multimedia sensor networks: A survey", Ain Shams Engineering Journal, 6, 481-490. 